\title{
A simulation study for fire extinguisher manufacturing process layout design
}

\author{
Ki-Young Jeong ${ }^{1 *}$, Jae-Dong Hong ${ }^{2}$ \\ ${ }^{1}$ Engineering Management, University of Houston Clear Lake, 2700 Bay Area Blvd, Houston, TX 77058, USA \\ ${ }^{2}$ Industrial Engineering Technology, South Carolina State University, 300 University Street NE Orangeburg, SC 29117, \\ USA
}

(Received March 6 2008, Revised May 24 2009, Accepted August 12 2009)

\begin{abstract}
This article provides a case study for a fire extinguisher manufacturing company in North America that has been building a new facility in Mexico to benefit from North America Free Trade Agreement (NAFTA) and low production cost. The company utilized a value stream mapping to finalize its new facility's manufacturing process design, and divided the process into several groups, some of which were being developed by contracted vendors. The powder fill operation was one of those vendor-developed systems. However its current layout design experienced the deadlock problem during the capacity test. To overcome this problem, the project team designed two other layouts - an express lane layout and an independent zone layout. The team used both an analytical approach and a simulation approach to evaluate all three alternatives including the current layout in terms of the number of pallets and capacity. The simulation study revealed that the independent zone layout (IZL) could satisfy the capacity requirement without any deadlock. However, the express lane layout (EZL), initially thought to be an alternative to the current layout, turned out to be infeasible. Through this case study, the team realized the importance and usefulness of the simulation-based design particularly when developing a new system. If it had been applied earlier before developing the actual system, it would have saved more time and cost. The team also realized that the diverse "what-if" analysis in the simulation could provide significant improvement in the design factors affecting the system performance.
\end{abstract}

Keywords: simulation-based design, capacity requirement pallets, critical pallets, layout design

\section{Background}

A company XYZ is a leading manufacturer of the portable fire extinguisher (PFE) in North America whose annual production capacity is around $8.5 \mathrm{M}$ units at two different facilities. The company has been building a new consolidated facility in Mexico to utilize the lower production cost and the tax benefits from North America Free Trade Agreement (NAFTA). The new facility construction was a challenging project. One of those challenges was to design the manufacturing process layout in a way that has enough production capacity to meet the perspective annual demand. The new facility construction project team adopted a value stream mapping (VSM) approach to design the manufacturing process layout. The VSM was selected based on the following reasons: (1) it supports easy and simple graphical symbols to capture the manufacturing processes $^{[3]}$; (2) it has a systematic mechanism to design a pull system by incorporating the takt time - average time between customer orders - and work-in-process (WIP) information for each process; (3) it could integrate both qualitative and quantitative information for an overall environment with those symbols and the mechanism ${ }^{[9]}$. The information includes scheduling policies, inventory replenishment policies, capacity, cycle time, lead time, and bottleneck information, etc. The project team initially developed several alternative process maps - all future state maps since they were for a new facility ${ }^{[7]}$, and eventually finalized them into one future state map. Based on the finalized VSM, the team divided the entire process into several groups,

\footnotetext{
* Corresponding author. Tel.: +1-281-283-3862, fax: 1-281-226-7590; E-mail address: jeongk@uhcl.edu.
} 
and made contracts with vendors for those groups. The contracted vendors were fully responsible for the contracted system assembly, integration, and a capacity test to ensure that the system satisfies the capacity requirement before it is shipped to the production site in Mexico. The capacity test requires vendors to operate the equipment and compare the time-between-productions to the takt time. If the time-between-productions is smaller than or equal to the takt time, the system is considered synchronized with the takt time, satisfying the capacity requirement. Otherwise the system should be revised.

A PFE mainly consists of a cylinder and a valve. The cylinder is made of either metal for industrial use or aluminum for residential use, and it contains the pressured dry chemical - powdered carbon dioxide (CO2). There are several different cylinder sizes whose diameter varies between 2.90 and 4.50 inches. The weight of the powder also varies between 1.35 and $6.00 \mathrm{lb}$ depending on the size of the cylinder. The manufacturing process of PFE mainly consists of (1) Cylinder Production and Test (CPT) - it forms a cylinder from an aluminum pack through a series of press machines, and checks if there is any crack; (2) Powder Fill Operation (PFO) - it fills the cylinder with the pressured chemical and the filling time could be slightly different for different cylinder types; (3) Torque and Charge Operation (TCO) - it controls the pressure inside the cylinder, and inserts the valve; (4) Final Assembly and Packaging (FAP) - it puts labels and small items such as a handle and a hose. It also includes the final packaging.

Through the VSM analysis, the project team identified that PFO and TCO were bottleneck processes. One vendor was developing an integrated PFO and TCO system on its site. The vendor partially completed its initial system layout. However, the team found out that the initial layout did not pass the capacity test since the system frequently halted even with a small number of cylinders, which triggered an active discussion on how to improve the initial system layout to satisfy the capacity requirement. Hence, solving this issue became an urgent business problem. Several alternative layouts were proposed through brainstorming, and eventually two of them were seriously considered. However, nobody was sure whether those proposed system layouts could satisfy the capacity requirement or not, and the team did not want to waste any more time and effort to modify the current system without ensuring that an alternative layout could satisfy the capacity requirement. The team also realized that there was no guarantee that the VSM-based future state map would be feasible since VSM highly abstracted the actual system without considering operational level verification and detailed factors such as interference between processes and system status change over time etc ${ }^{[2,9]}$.

To overcome this gap between the VSM and the actual system layout, and to minimize the time loss associated with frequent modifications, the project team decided to use simulation and an analytical approach if any to evaluate alternatives. Simulation is a powerful tool to solve a complex system design problem without resorting to complex analytical forms if important system parameters are identified ${ }^{[8]}$. Another advantage of simulation - particularly for this case study - is that it provides the diverse "what-if" scenario-based analysis. It would be ideal if analysts could identify all important system design factors which affect the system performance before actual implementation. However, it would be often very difficult to define all those factors in advance. Instead, many factors are clearer when more information for a system is obtained as time passes. This concept is called the progressive elaboration in a project management community ${ }^{[10]}$. Schwalbe ${ }^{[1]}$ considered the progressive elaboration as one of the project attributes, and claimed that a project should be developed in increments to accommodate this information update. Similarly, as more system knowledge becomes available through the "what-if" scenario analysis, the team could identify more accurate system design factors in this case study. However, the significant effort is required to develop a right simulation model that well represents the real system. That is why the team considered both an analytical approach and simulation together.

The remainder of this paper is organized as follows: Current Layout section provides general information about the current layout and related operational problems, followed by Layout Alternatives section showing two alternative layouts. The concepts of the optimal design with maximum throughput with minimum number of pallets are discussed in Critical Pallets and Capacity Requirement Pallets section, and the analytical approach is discussed in Analytical Approaches for CRP section where the number of pallets required to satisfy the target throughput is derived for each alternative layout. Simulation and Results section provides the detailed simulation analysis and evaluation of each layout, followed by Discussions section showing the comparison between the analytical approach and simulation approach using the independent zone layout as a testbed, followed by Conclusion section. 


\section{Current layout}

As discussed earlier, A PFE is manufactured through four processes, which are connected through belt conveyors on which pallets are transported. Once a cylinder finishes CPT, it is delivered to PFO and loaded on a pallet at a loading machine, and travels through the system. Currently, both PFO system (PFOS) and TCO system (TCOS) have its own pallet group, and no pallet is shared between these two groups. In PFOS, an empty cylinder on a pallet is filled with dry chemical powder, and this loaded pallet is delivered to TCOS where a valve is inserted and the pressure of a cylinder is controlled for proper discharge. The current layout (CL) of the PFO and TCO is shown in Fig. 1.

The rectangle in the figure represents a machine, and the solid line between the machines represents the conveyor belt. For example, the PFOS has two loading machines (L) where an unloaded pallet is loaded with an empty cylinder and its total weight is measured at one of pre-weighing machines (PW). As seen in the figure, PFOS consists of five powder fill stations (PFS) where each PFS consists of multiple lanes of a powder fill machine (F) and a post-weighing machine (W). The pallet loaded with an empty cylinder is sent to one of five PFSs according to a production schedule. The first two stations have four parallel lanes while the last three stations have six parallel lanes. The TCOS consists of five TC stations (TCS), each of which is also connected by the conveyor belt with a corresponding PFS. The first two TCSs have one valve insertion machine (V) and two parallel torque and charge machines (T) while the last three TCSs have one value insertion machine (V) and three parallel torque and charge machines. Note that the place where PFS and TCS meet is called a junction (J) or a pick and place where a robot arm picks a filled cylinder from a pallet in PFS and moves it onto an unloaded pallet in TCS.

The operation sequence of this entire system can be explained as follows: Pallets are circulating on the conveyor belt in PFOS and TCOS. Whenever an unloaded pallet passes through the loading machines (L), the pallet is loaded with a specific empty cylinder which is supplied outside. The weight of the loaded pallet is then measured on a pre-weighing machine (PW), and the loaded pallet is assigned to one of the available powder fill machines (F) specified by the production schedule. Once a cylinder on a pallet is filled at a powder fill machine, its weight is measured on a post-weighing machine (W), and it resumes its travel along the conveyor belt. When it reaches a junction $(\mathrm{J})$, the filled cylinder is picked up from the pallet, and transferred by a robot arm onto an unloaded pallet available in a corresponding TCS. That is, a pallet-switching occurs. Then the unloaded pallet at the junction continues to move toward the loading machines again in PFOS. The loaded pallet in the TCS circulates counterclockwise, and goes through a valve insertion machine (V) and one of the torque and charge machines $(\mathrm{T})$. Once both machines finish their operations, the cylinder is transferred into the next Final Assembly and Packaging (FAP) station at the transfer machine denoted by a circle with " $x$ " in Fig. 1, and the unloaded pallet continues its travel toward the junction to pick up another filled cylinder from PFOS. In summary, in the current layout, there are two closed loops of pallet movement: pallets circulating clockwise in PFOS, and pallets circulating counterclockwise in TCOS. The pallet switching between these two loops occurs at the junction where two operation stations meet.

The vender performed the capacity test with only three PFSs and TCSs. However, the project team noticed that the entire system frequently halted due to the heavy pallet accumulation on the returning segment of the conveyor belt between loading machines and junctions. From now on, this segment of a conveyor belt is called as the returning conveyor belt segment (RCBS). Note that both loaded pallets and unloaded pallets share the RCBS. For example, consider the RCBS connecting the junction in the station 3 in PFOS and the loading machines. Unloaded pallets are coming from all five PFO stations, and loaded pallets are coming from the first two PFO stations before they are transferred into TCOS. Since both types of pallets are mixed and moving together, this caused severe congestion and delay on the RCBS. Of course, the most congested area was the RCBS between loading machines and the junction in the first PFO station since all pallets have to move through that segment. Another important observation was that the pick and place operated by a robot arm exacerbated the congestion because while the filled cylinder was transferred from PFS to a corresponding TCS, all incoming pallets including both loaded and unloaded pallets should stop and wait until this operation finishes. More specifically, when the heavy pallet accumulation occurs on RCBS, all space available on that RCBS is consumed up, which eventually creates a deadlock by blocking pallet movements. A deadlock is defined as a situation where two or more competing actions are waiting for the other to finish, and thus neither 


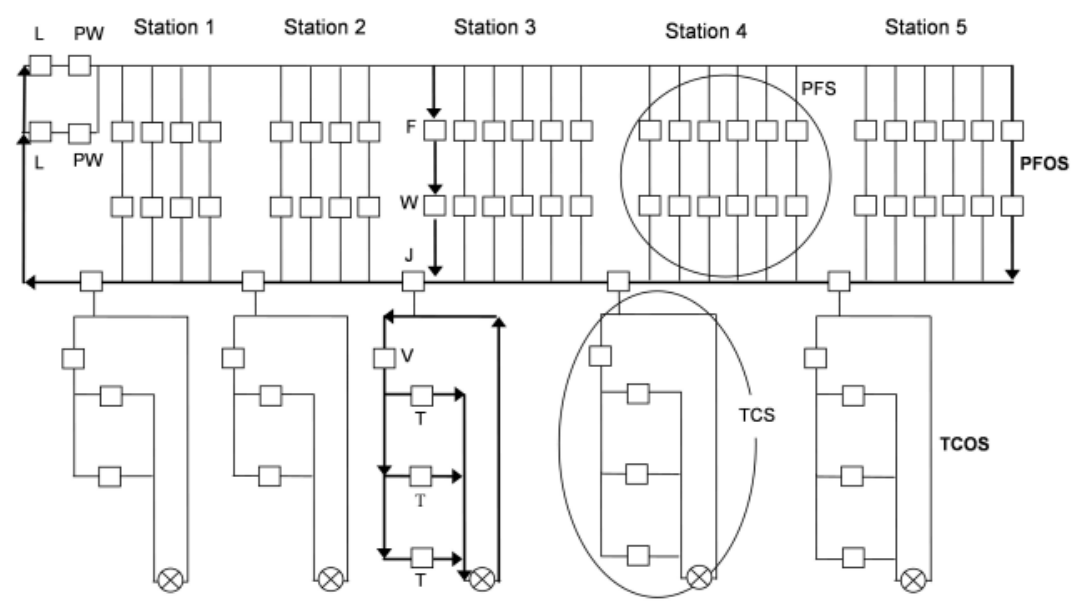

Fig. 1. Current PFO and TC Layout (CL)

ever does. For example, if all space on RCBS in front of the first station is used up and if loading machines send unloaded pallets to the first PFS, the deadlock occurs when all space available at the first PFS is occupied. The project team performed several capacity requirement tests with different numbers of pallets since the number of pallets apparently affected the congestion level. However, they could not figure out how many pallets were required to make the current system feasible. They were not even sure whether the current layout could be feasible - satisfying demand - or not.

\section{Layout alternatives}

To solve this congestion and deadlock issue, the team proposed two other alternatives - an express lane layout (ELL) and an independent zone layout (IZL) - through brainstorming. However, the team felt tight time pressure because of the pending delivery due date - only three months left. Fortunately, the mechanical structure of PFOS and TCOS makes flexible assembly and disassembly possible. Hence, if a final feasible alternative layout is decided, the team estimated that it would take approximately two months to reconfigure the system with all tests.

\subsection{Express lane layout}

This alternative was to add a dedicated express lane to manipulate unloaded pallets coming from the TCOS. The overall layout is depicted in Fig. 2. The operation sequence can be explained as follows: Whenever an unloaded pallet passes through loading machines (L), the pallet is loaded with a specific cylinder based on the production schedule. The weight of the loaded pallet is then measured on a pre-weighing machine (PW), and the loaded pallet travels to one of the available powder fill machines (F). Once the cylinder on the pallet is filled, its weight is measured on the post-weighing machine (W) - note that now a PFS has one shared post-weighing machine. Then, without pallet switching, the loaded pallet with a filled cylinder travels to the corresponding TCS. Once the cylinder finishes its operation at TCS, it is transferred to FAP, and the unloaded pallet moves to loading machines using the express lane. They expected this express lane layout could alleviate the congestion caused by the mixture of the loaded pallets and unloaded pallets, reducing the chance of the system halt. Note that there is a junction where a PFS meets a TCS. However, the pick and place operation is not performed any more in this layout. In other words, this junction works only as a connection point between PFOS and TCOS.

\subsection{Independent zone layout}

Another alternative used the zone control concept where an express lane layout was replaced by five independent zones where each zone consists of a single PFS and a single TCS with its own loading machine. Hence, there is no interaction between zones. This was called an independent zone layout (IZL), and is 


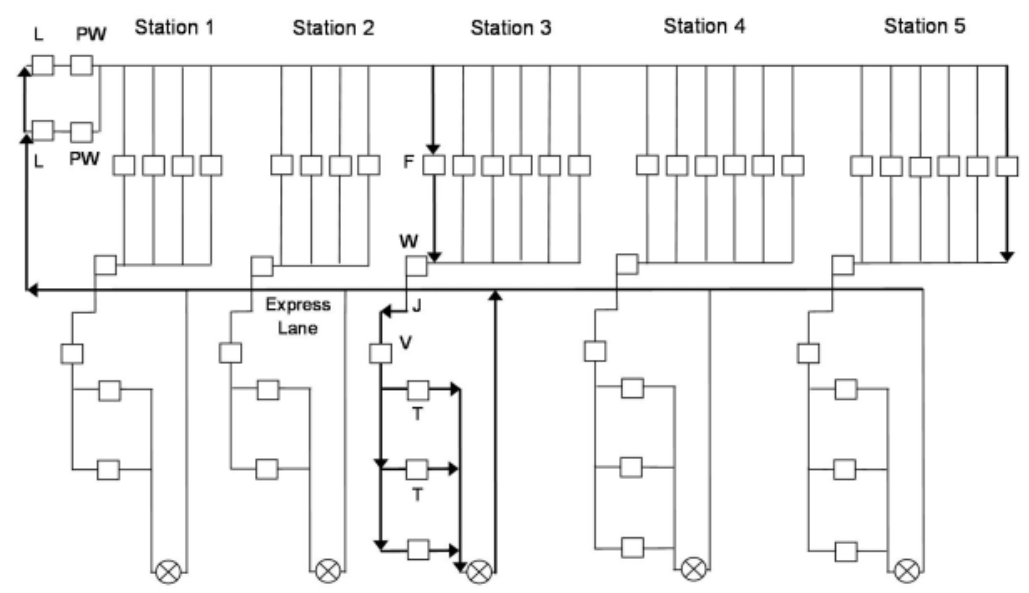

Fig. 2. Express Lane Layout (ELL)

depicted in Fig. 3. The rationale behind this was that it could simplify the traffic by eliminating intervention between zones, and avoid any potential control and collision problems. It could also contribute to the balanced pallet distribution - 'balanced' means the equal distance between pallets on the belt conveyor ${ }^{[5]}$.
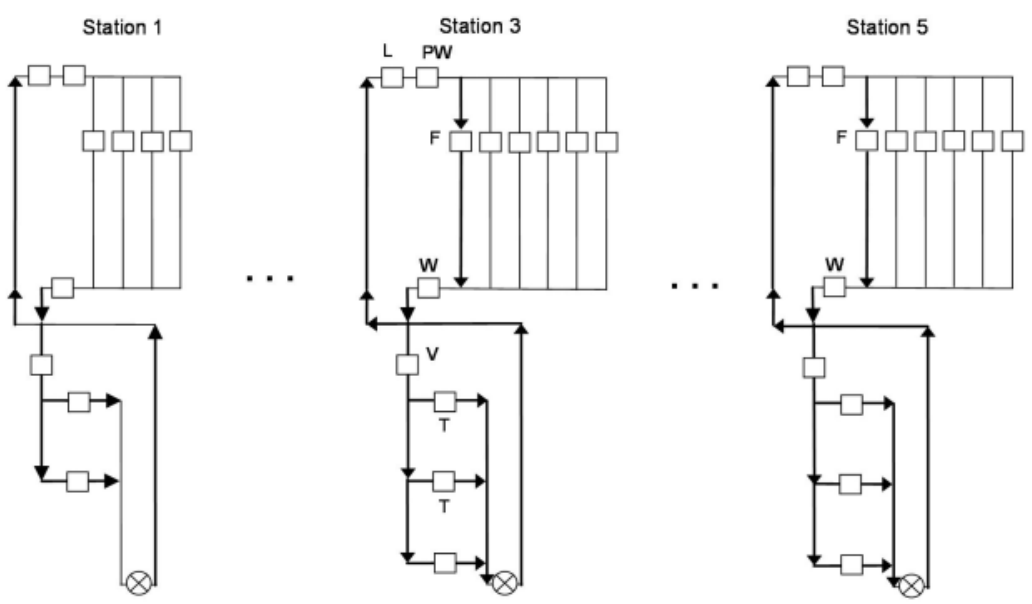

Fig. 3. Independent Zone Layout (IZL)

Many expected that the express lane layout could release the congestion to some extent. However, they were also concerned about the side effect of the longer pallet travel distance introduced in this design - note that a pallet travels all the way between PFOS and TCOS - since it could impair the balanced pallet distribution as described in Tab. 1 where three alternatives were compared in terms of their characteristics and potential issues. Many also agreed that the IZL could have maximum capacity, but it would require more space and layout change. However, nobody was still sure of its capacity and feasibility. It would be interesting to note that the current layout also used the zone concept by separating two different pallet groups at PFOS and TCOS. However, this zone concept is eliminated in the express lane layout to expedite the returning pallet movement. Hence the advantages and disadvantages of this layout change should be evaluated. For all three layouts, it should be also recognized that the capacity needs to be measured and it is closely related to the number of pallets on the layout.

\section{Critical pallets and capacity requirement pallets}

To fill the gap between VSM analysis and the actual implementation, the team needed to answer the following questions before taking any action for further modification works. 
Table 1. Preliminary Comparison

\begin{tabular}{l|l|l}
\hline \multicolumn{1}{c|}{ Layout } & \multicolumn{1}{|c}{ Characteristics } & \multicolumn{1}{c}{ Potential Issues } \\
\hline $\begin{array}{l}\text { Current } \\
\text { Layout }\end{array}$ & $\begin{array}{l}\text { (1) Two shared loading stations feed entire lines } \\
\text { (2) A pallet switching occurs between PFS and TCS } \\
\text { (3) Loaded pallets and unloaded pallets share the same } \\
\text { conveyor belt to return to the loading machines }\end{array}$ & $\begin{array}{l}\text { (1) Severe congestion on the shared conveyor } \\
\text { belt observed, which created the deadlock } \\
\text { (2) The pick and place could block pallet flow }\end{array}$ \\
\hline $\begin{array}{l}\text { Express } \\
\text { Lane Layout }\end{array}$ & $\begin{array}{l}\text { (1) The same pallet travels from PFS to TCS without } \\
\text { any pallet switching } \\
\text { (2) The express lane is separately added for unloaded } \\
\text { pallet transportation from TCS to loading machines }\end{array}$ & $\begin{array}{l}\text { (1) Longer pallet travel distance } \\
\text { may impair the balanced pallet distribution, } \\
\text { which could exacerbate the congestion }\end{array}$ \\
\hline Independent & $\begin{array}{l}\text { (1) Each zone works independently with its own } \\
\text { loading machine. } \\
\text { Zone Layout Each station works like an express lane layout with } \\
\text { its own loading } \\
\text { machine }\end{array}$ & $\begin{array}{l}\text { (1) More time and effort to build the system } \\
\text { (2) More space required }\end{array}$ \\
\hline
\end{tabular}

(1) Which layout could satisfy the capacity requirement?

(2) How many pallets are required to optimize the performance of the layout?

These questions will be discussed in detail in the next few sections. Before going further, it is important to recognize that these two questions are interrelated. For example, a small number of pallets may be helpful to alleviate the congestion. However, too small number of pallets could prevent the system from achieving the capacity requirement - e.g., annual target production quantity (or annual throughput). As the number of pallets increases, the throughput increases without delay in the pallet transportation time from origin to destination - this time is called the cycle time. This trend between throughput and the number of pallets continues until the number of pallets reaches a threshold value. Even though the number of pallets is increased beyond the threshold value, the throughput does not increase any more since the congestion creates delay in pallet movements. Hence, throughput is a non-decreasing function of the number of pallets if no deadlock occurs. In general, the number of work-in-process (WIP) - pallets in this case study - at the threshold value is called a critical WIP (or a critical pallet). In other words, two questions could be answered if the critical pallet (CP) where the system throughput is maximized is identified. Readers are encouraged to refer to [6] to understand the relationship among WIP, throughput and cycle time.

To find $\mathrm{CP}$ is an important issue since it could maximize the system throughput with the minimum number of pallets. However, due to the interaction caused by the complex layout, the development of an analytical approach for CP could be a complex problem. That is why simulation could be useful. However, even though $\mathrm{CP}$ is found with simulation, there is no guarantee that the throughput at $\mathrm{CP}$ could satisfy the target throughput. Hence, the throughput at $\mathrm{CP}$ should be compared with the target throughput. If the throughput at $\mathrm{CP}$ does satisfy the target throughput, the system layout is considered feasible. Otherwise, it does not need to be considered further.

Fig. 4 provides how to find out CP in simulation. Initially, simulation starts with very small number of pallets stored in NP (step 2) after initialization (step 1). Once the model generates a throughput (step 3), the throughput from simulation (SimTP) is considered as the current throughput (CurTP) in step 4. If the current throughput and the previous throughput (PreTP) are identical (step 5), it means that simulation reaches CP, and the system feasibility is evaluated by comparing the current throughput with the target throughput (step 8). If the current throughput is larger than or equal to the target throughput, it means that current layout is feasible (step 9). Otherwise, it means that the current layout could not satisfy the capacity requirement (step 10). If CurTP and PrevTP are not identical in step 5, it needs to consider the current throughput as the previous throughput (step 6), and increases the number of pallets by (step 7), and reruns the model (step 3). Note that the algorithm in Fig. 4 utilizes the rationale that throughput is a nondecreasing function of WIP.

The capacity requirement pallet (CRP) is the number of pallets required to meet the capacity requirement for each layout. If CRP is smaller than CP, the CRP is the minimal number of pallets required to satisfy the demand. The relation between CP and CRP could provide many useful insights on the system, and CRP could be analytically computed without time consuming simulation. Later CRP would be evaluated with simulation 
to see whether it is feasible or not under more realistic conditions. As discussed in detail in the next section, the CRP is estimated based on several simplifying assumptions without full consideration of complexities complex topology and structure - founded in the actual layouts. Therefore, the gap between analytical CRP and its simulation-based solution could be interpreted as the impact of the topology and layout structure.

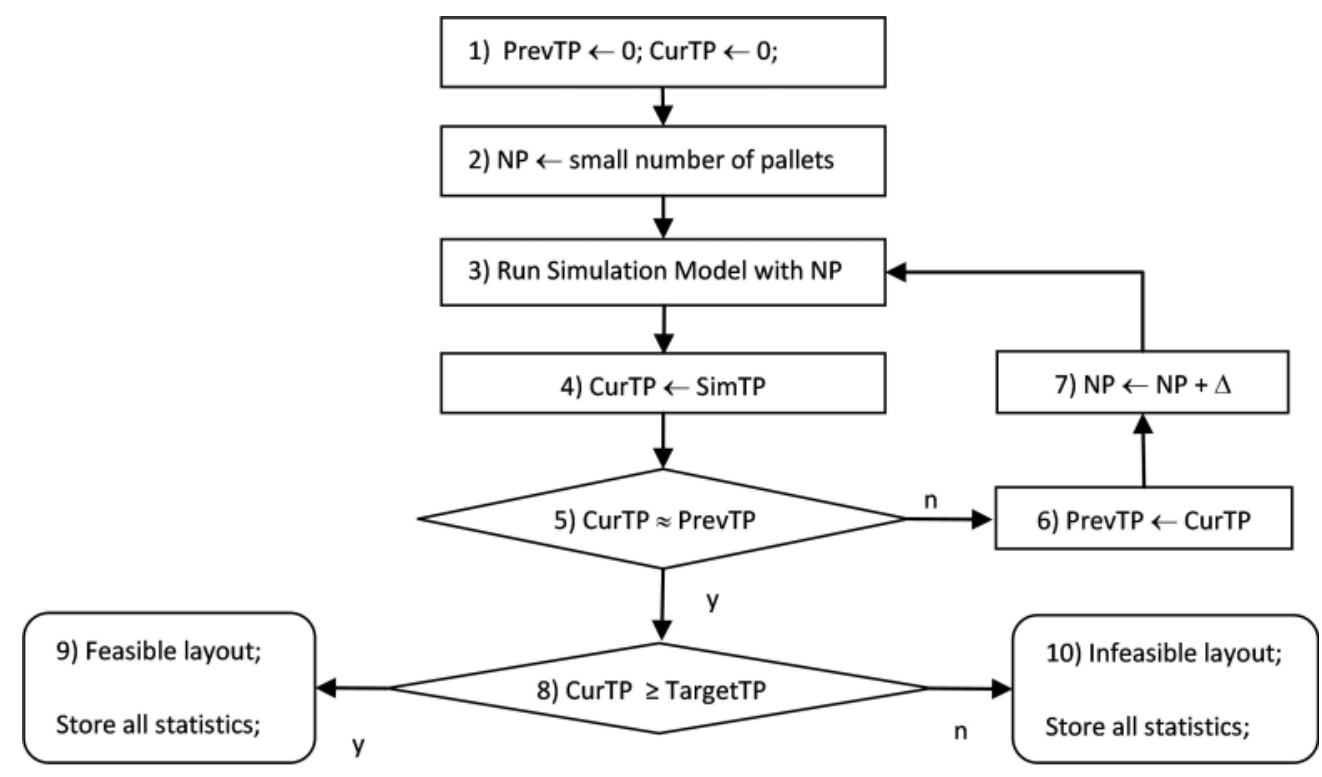

Fig. 4. Simulation-Based CP Computation

\section{Analytical approaches for CRP}

In this section, the analytical approach is described to compute CRP for each layout. The approach starts with the target throughput - number of completed cylinders per time - and it computes the delivery cycle time time required for a pallet to circulate from a loading machine to a loading machine. The number of completed cylinders per time is approximated by the number of pallets delivered per time since one pallet can deliver only one cylinder, and these two numbers should be equivalent in the steady state. The multiplication of the target throughput rate and the delivery cycle time provides the number of pallets required to meet the target throughput. The overall approach in this section is analogous to the computation of the number of Automated Guided Vehicles required in the material handling system ${ }^{[4]}$. The detailed discussion is provided below.

\subsection{Analysis of current layout}

The CRP in the current layout (CL) is attempted first. Using Fig. 1, the following notations are defined, and the two closed loops of pallets are analyzed individually.
$p_{j}:$
probability that a pallet starts from $j^{\text {th }}$ loading machine, $j=1,2$
$q_{k}: \quad$ probability that a pallet returns to $k^{\text {th }}$ loading machine in PFOS, $k=1,2$
$r_{s}: \quad \quad \quad \quad$ probability that $s^{\text {th }}$ station is selected in PFOS where $s=\{1,2,3,4,5\}$
$u_{i}^{s}$ : $\quad$ probability that $i^{t h}$ lane is selected in $s^{\text {th }}$ PFS where $i=1,2,3,4$ for stations 1 and 2, $i=\{1,2,3,4,5,6\}$ for remaining stations.
$v: \quad$ velocity of a pallet defined by the velocity of the conveyor.
$L_{s}^{i}(j, k)$ : total travel distance of a pallet when $i^{\text {th }}$ lane in $s^{\text {th }}$ PFS is selected, given that the pallet starts from $j^{\text {th }}$ loading machine, and returns to $k^{\text {th }}$ loading machine. 
$O_{s}^{i}(j, k)$ : total sum of operation times for a pallet when a pallet uses $i^{\text {th }}$ lane in $s^{\text {th }}$ PFS given that it starts from $j^{\text {th }}$ loading machine, and returns to $k^{\text {th }}$ loading machine.

$d_{P F O}: \quad$ target delivery rate in the PFOS, which is defined by the number of cylinders loaded per time. The two nine-hour shifts for 52 weeks per year are assumed.

Then, the average ideal delivery cycle time of a pallet in PFOS, denoted by $T_{P F O}$, is defined by

$$
T_{P F O}=\sum_{j} \sum_{s} \sum_{i} \sum_{k} p_{j} r_{s} u_{s}^{i} q_{k}\left\{\frac{L_{s}^{i}(j, k)}{v}+O_{s}^{i}(j, k)\right\}
$$

Then, the number of pallets required, $N_{P F O}$, to meet the target delivery rate $d_{P F O}$ is computed by

$$
N_{P F O}=d_{P F O} T_{P F O}
$$

However, it is important to recognize that the Eq. (1) and (2) simplify many realities. First, they do not consider any factor which makes the pallet movement slow such as traffic congestion, and topology and mechanical structure of the system. They also assume that an unloaded pallet at TCS is always available whenever a cylinder finishes its operation at PFOS. In practice, this assumption may be realized by adding enough number of pallets to TCS. If the congestion caused by the topology is not severe, the solution from Eq. (2) could be too small to realize the target throughput. However, if there is significant congestion and potential deadlock caused by the system topology, the solution could be too large. In the latter case, the number of pallets should be reduced to make the system less congested. It should be also emphasized that only one cylinder type is assumed for this capacity analysis since all different cylinder types had the same process and their processing times were not significantly different.

The following data and constraints were used to compute the CRP in this case study.

- Total number of target production quantity $=8.5 \mathrm{M}$

- $\quad$ Total time per year $=(2$ shifts $)(9$ working hours $/$ shift $)(5$ days $/$ week $)(52$ weeks $/ y r)=280,800 \mathrm{~min}$

Hence, the target throughput, $d_{P F O} \cong 31$ units/min. Each loading machine and a path had an equal chance to be selected. Hence

- $\quad p_{j}=1 / 2$ for $j=1,2$

- $\quad u_{i}^{s}=1 / 4$ for $s=1,2$ for $i=1, \cdots, 4 ; p_{i}^{s}=1 / 6$ for $s=3,4,5$ for $i=1, \cdots, 6$

- $\quad q_{k}=1 / 2$ for $k=1,2 ; q_{k}=1 / 3$ for $k=3,4,5$

It was assumed that the station selection probability was proportional to the number of lanes in a station. Hence

$r_{s}=4 / 26$ for $s=1,2 ; r_{s}=6 / 26$ for $s=3,4,5$

The speed for conveyor was set as $v=0.5 \mathrm{inch} / \mathrm{sec}$, and the average time information was given at Tab. 2.

Based on the probabilities and time information, all possible travel paths should be analyzed in Eq. (1) to compute $T_{P F O}$, which was $2.99 \mathrm{~min}$. The similar approach could be applied to TCOS. The metrics in the current layout were summarized in Tab. 3. The total number of pallets is the sum of all pallets in both PFOS and TCOS.

\subsection{Analysis of express lane layout}

Based on Fig. 2, a similar procedure could be applied to the express lane layout (ELL). A loaded pallet starting from a loading machine in PFOS travels all the way down to TCOS without pallet switching. Once a cylinder is delivered to the next FAP in TCOS, the unloaded pallet travels to one of loading machines through the express lane. Hence, there is only one closed loop of pallet movement. The following notations are additionally introduced to accommodate the pallet movement in TCOS.

$h_{s}^{l}: \quad \quad \quad$ probability that $l^{t h}$ lane is selected in $s^{t h}$ TCS.

$L_{s}^{i}(j, l, k)$ : total travel distance of a pallet when $i^{t h}$ lane in $s^{\text {th }}$ PFS is selected, and the pallet starts from $j^{\text {th }}$ loading machine, and returns to $k^{\text {th }}$ loading machine through $l^{\text {th }}$ lane in TCS.

$O_{s}^{i}(j, k, l): \quad$ total sum of operation times for a pallet when $i^{t h}$ lane in $s^{\text {th }}$ PFS is selected, and the pallet starts from $j^{t h}$ loading machine, and returns to $k^{\text {th }}$ loading machine through $l^{\text {th }}$ lane in TCS. 
Table 2. Please write your table caption here

\begin{tabular}{l|ccc}
\hline Operations & Time (sec) & $\begin{array}{l}\text { Cumulative time } \\
\text { for PFO }\end{array}$ & $\begin{array}{l}\text { Cumulative time } \\
\text { for TCO }\end{array}$ \\
\hline Loading & 4 & 4 & \\
Pre-weigh & 4 & 8 & \\
Post-weigh & 4 & 12 & \\
PFO & 25 & 37 & \\
Pick \& Place & 3.5 & 40.5 & 7.5 \\
Valve insertion & 7.5 & & 22.5 \\
TC processing & 15 & & 27.5 \\
Unloading at TC & 5 & & \\
\hline
\end{tabular}

Table 3. CRP in Current Layout

\begin{tabular}{ll}
\hline \multicolumn{1}{c}{ Item } & Values \\
\hline$d_{P F O}$ & 31 units/min \\
$T_{P F O}$ & 2.99 min \\
$N_{P F O}$ & 92 pallets \\
$N_{T C O}$ for stations 1 and 2 & 7 pallets \\
$N_{T C O}$ for stations 3, 4, and 5 & 9 pallets \\
Total Pallets & 133 pallets \\
\hline
\end{tabular}

Then, the average delivery cycle time per delivery of a pallet, denoted by $T_{P F O / T C O}$, is defined by

$$
T_{P F O / T C O}=\sum_{j} \sum_{s} \sum_{i} \sum_{l} \sum_{k} p_{j} r_{s} u_{s}^{i} h_{s}^{l} q_{k}\left\{\frac{L_{s}^{i}(j, l, k)}{v}+O_{s}^{i}(j, l, k)\right\}
$$

Once the delivery cycle time, $T_{P F O / T C O}$, was computed by Eq. (3), which was $4.39 \mathrm{~min}$, the CRP, $N_{P F O / T C O}$, was computed by Eq. (2) again, and the results were summarized in Tab. 4.

As seen in Tab. 3 and Tab. 4, there is no significant difference in the required number of pallets between two layouts. This is because two layouts are almost identical in terms of the topology except the express lane.

\subsection{Analysis of independent zone layout}

The independent zone layout (IZL) would be designed in a way that splits the ELL into five independent zones, each of which has its own single loading machine as seen in Fig. 3. The zones 1 and 2 are identical while remaining three zones are identical. Hence, the Eq. (3) can be modified into the simpler form as in Eq. (4) since it has only one loading and unloading machine, respectively. For each $s^{\text {th }}$ independent zone, the average delivery cycle time per delivery of a pallet in PFOS and TCOS stations, denoted by $T_{P F O / T C O}$, is defined by

$$
T_{P F O / T C O}=\sum_{i} \sum_{j} u_{s}^{i} h_{s}^{j}\left\{\frac{L_{s}(i, j)}{v}+O_{s}(i, j)\right\}
$$

where $L_{s}(i, j)$ is the total travel distance of a pallet when $i^{\text {th }}$ lane in PFS is selected, and the pallet returns to the loading machine through $j^{\text {th }}$ lane in TCS, and $O_{s}(i, j)$ represents the corresponding sum of operation times on those lanes selected.

$\mathrm{TPFO} / \mathrm{TCO}=2.89 \mathrm{~min}$ for the first two zones and $T_{P F O / T C O}=3.09 \mathrm{~min}$ for the last three zones were computed. It was assumed that the target delivery rate, $d_{P F O / T C O}=31$ units $/ \mathrm{min}$, was allocated to each independent zone in proportion to the number of lanes in PFS in the zone - The problem of this assumption will be discussed later. For example, for the first two zones, the delivery rate was computed to $d_{P F O / T C O}=(31$ units $/ \mathrm{min}) \times(4 / 26)=4.73$ units $/ \mathrm{min}$. Hence $N_{P F O} / T C O$ was estimated at $4.73 \times 2.89 \cong 14$ pallets. The results for each zone were summarized in Tab. 5 .

Compared to other layouts, the IZL significantly reduced the number of pallets through the zone concept. 
Table 4. CRP in Express Lane Layout

\begin{tabular}{ll}
\hline \multicolumn{1}{c}{ Item } & \multicolumn{1}{c}{ Values } \\
\hline$d_{P F O / T C O}$ & 31 units/min \\
$T_{P F O / T C O}$ & 4.39 min \\
$N_{P F O / T C O}$ & 135 pallets \\
Total & 135 pallets \\
\hline
\end{tabular}

Table 5. CRP in Independent Zone Layout

\begin{tabular}{cc}
\hline Item & Values \\
\hline \multicolumn{2}{c}{ For stations 1 and 2} \\
\hline$d_{P F O / T C O}$ & 4.73 units/min \\
$T_{P F O / T C O}$ & 2.89 min \\
$N_{P F O / T C O}$ & 14 pallets \\
\hline \multicolumn{2}{c}{ For stations 3,4, and 5 } \\
\hline$d_{P F O / T C O}$ & 7.10 units/min \\
$T_{P F O / T C O}$ & 3.09 min \\
$N_{P F O / T C O}$ & 22 pallets \\
\hline Total & 94 pallets \\
\hline
\end{tabular}

\section{Simulation and results}

Once the analytical solutions for CRP were obtained, simulation was used to evaluate them. The simulation was also used to estimate CP. It is important to recognize that the simulation could be used to improve detailed design factors which could be identified through the progressive elaboration with "what-if" scenario analysis. Specifically speaking, instead of developing three independent simulation models to evaluate three alternatives, one simulation model could be built first for a specific layout. Then the design factors identified from this simulation model could be improved and modified, and they could be used at other simulation models. For example, the simulation model for IZL was developed after the several experiments had been tried with the ELL model to overcome the limitations found in ELL.

Flexsim simulation software was used since it provided very realistic and convenient 3D visualization and the conveyor belt design and control mechanism ${ }^{[1]}$. Since the operation time was very stable, the uniform distribution with $2.5 \%$ variations was considered enough. All simulations were run for one year length run time with one month warm up period, and repeated five times. Since the evaluation of the topology and design structure was a main issue, it was assumed that the model had no machine breakdown. Based on these, the results were summarized in Tab. 6 . The first column shows the case number, and the second column shows the primary variables: three layout types - CL, ELL, and IZL - and two schedule types - schedule 1 and schedule 2 . The sched 1 is a current scheduling method where pallets are proportionally distributed to PFS according to the number of lanes in the station, $(4 / 24,4 / 26,6 / 26,6 / 26,6 / 26)$, while the sched 2 is a new scheduling method where less pallets are assigned to shorter distanced PFSs from the loading machines while more pallets are assigned to longer distanced PFSs to reduce the congestion on RCBS. In this experiment, the sched 2 was set to $(1 / 26,2 / 26,7 / 26,7 / 26,9 / 26)$. The third column shows the annual throughput at different numbers of pallets where the cell with "X" implies that the combination of three factors - a layout type, a scheduling method, and the number of pallets - created the deadlock, which created the system halt. From this table, the following phenomena are directly observed.

- The computed CRP in both CL and ELL created a system halt. As seen in the first case, the CL worked with only 75 pallets. That is, the CL was not a feasible layout since it could not satisfy the capacity requirement. The same was true in the ELL

- By comparing the Case 1 with the Case 2, the scheduling method was not a major factor to avoid the system halt although it contributed to reducing the system halt to some extent.

- The ELL (Case 3-1) performed even worse than CL. This naturally created a "why" question, and the answer was very simple but critical. The junction between PFS and TCS created a severe deadlock since it was shared by PFOS and TCOS. When one of those junctions was blocked, the entire express lane was blocked as seen in the screen shot of Flexsim in Fig. 5.

- Based on the progressive elaboration concept, the issue with the junction identified in Figure 5 was examined, and it was eliminated in the way that makes a pallet from PFOS travel to TCOS underneath of the express lane. This was realized by an escalator-like structure, and this added extra three seconds to the pallet movements. This new design was applied to both ELL and IZL designs. However, the results from the new ELL (Case 3-2) were still not feasible. The results from IZL (Case 4) were also obtained using this design factor improvement. 
Table 6. Experiments at different conditions

\begin{tabular}{|c|c|c|c|c|c|c|c|c|c|c|c|c|c|}
\hline \multirow{2}{*}{$\frac{\text { CASE }}{1}$} & \multirow{2}{*}{$\begin{array}{l}\text { VARI- } \\
\text {-ABLES } \\
\text { CL } \\
\text { Sched } 1\end{array}$} & \multicolumn{12}{|c|}{ Annual Throughput at Different Total Numbers of Pallets } \\
\hline & & 175 & 165 & 155 & 145 & 135 & 125 & 115 & 95 & 85 & 75 & & \\
\hline \multirow{3}{*}{2} & \multirow{3}{*}{$\begin{array}{l}\text { CL } \\
\text { Sched } 2\end{array}$} & $\mathrm{X}$ & $\mathrm{X}$ & $\mathrm{X}$ & $X$ & $X$ & $X$ & $\mathrm{X}$ & $\mathrm{X}$ & $\mathrm{X}$ & $3.5 \mathrm{M}$ & & \\
\hline & & 175 & 165 & 155 & 145 & 135 & 125 & 115 & 95 & 85 & 75 & 65 & 55 \\
\hline & & $\mathrm{X}$ & $X$ & $X$ & $X$ & $X$ & $\mathrm{X}$ & $3.7 \mathrm{M}$ & $3.7 \mathrm{M}$ & $3.7 \mathrm{M}$ & $3.7 \mathrm{M}$ & $3.7 \mathrm{M}$ & $2.5 \mathrm{M}$ \\
\hline \multirow[t]{2}{*}{$3-1$} & \multirow[t]{2}{*}{$\begin{array}{l}\text { ELL } \\
\text { Sched } 1\end{array}$} & 175 & 165 & 155 & 145 & 135 & 125 & 115 & 95 & 85 & 75 & 65 & 55 \\
\hline & & $\mathrm{X}$ & $\mathrm{X}$ & $\mathrm{X}$ & $\mathrm{X}$ & $X$ & $X$ & $\mathrm{X}$ & $X$ & $1.6 \mathrm{M}$ & $1.6 \mathrm{M}$ & $1.6 \mathrm{M}$ & $1.6 \mathrm{M}$ \\
\hline \multirow[t]{2}{*}{$3-2$} & \multirow[t]{2}{*}{$\begin{array}{l}\text { ELL } \\
\text { Sched } 1\end{array}$} & 175 & 165 & 155 & 145 & 135 & 125 & 115 & 95 & 85 & 75 & 65 & 55 \\
\hline & & $\mathrm{X}$ & $X$ & $\mathrm{X}$ & $\mathrm{X}$ & $X$ & $6.2 \mathrm{M}$ & $6.2 \mathrm{M}$ & $6.2 \mathrm{M}$ & $5.8 \mathrm{M}$ & $5.5 \mathrm{M}$ & $5 \mathrm{M}$ & $4 \mathrm{M}$ \\
\hline \multirow[t]{2}{*}{4} & \multirow[t]{2}{*}{$\begin{array}{l}\text { IZL } \\
\text { Sched } 1\end{array}$} & 175 & 165 & 140 & 135 & 125 & 115 & 105 & 90 & 75 & & & \\
\hline & & $X$ & $\mathrm{X}$ & $10.5 \mathrm{M}$ & $10.5 \mathrm{M}$ & $10.5 \mathrm{M}$ & $10.5 \mathrm{M}$ & $10.5 \mathrm{M}$ & $10.5 \mathrm{M}$ & $9 \mathrm{M}$ & & & \\
\hline
\end{tabular}

- It was found that the computed CRP - 94 pallets - for IZL was feasible without any system halt, and CRP was larger than $\mathrm{CP}$ - 90 pallets. The $\mathrm{CP}$ also satisfied the capacity requirement. According to Tab. 6 , even 75 pallets could satisfy the capacity requirement.

- The IZL could produce up to $10.5 \mathrm{M}$, more than the capacity requirement. From managerial perspectives, the facility could use this extra $2 \mathrm{M}$ surplus capacity as the buffer for demand fluctuation and machine breakdown.

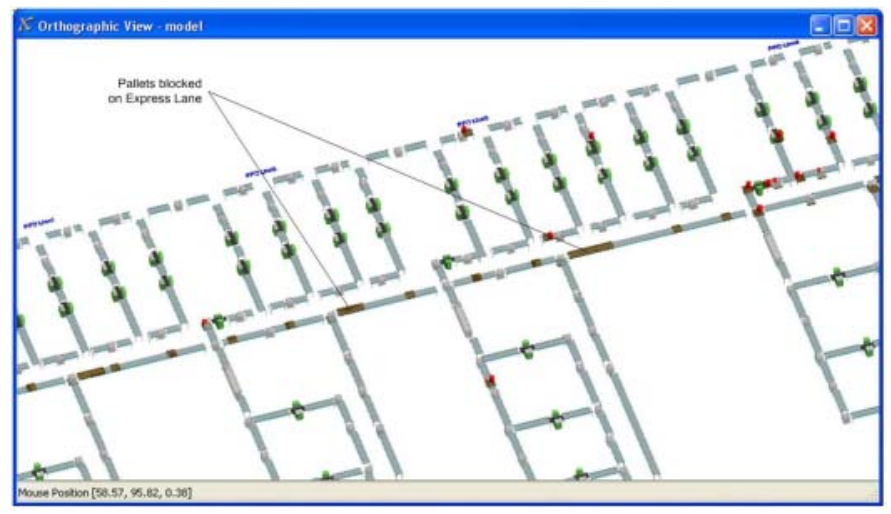

Fig. 5. Screen shot of ELL Simulation

Fig. 6 shows detailed simulation-based CP analysis for two different independent zones at IZL. The x-axis and y-axis show the number of pallets and the annual throughput - note that the unit is million - respectively. The $\mathrm{CP}$ at the four-lane zone is around 15 and it could produce up to $1.9 \mathrm{M}$ while the $\mathrm{CP}$ at a six-lane zone is around 20 , and it could produce up to $2.3 \mathrm{M}$. It is interesting to see that when the number of pallets is small (e.g. less than 15), the four-lane zone could produce more than the six-lane zone. The CP and its throughput in Fig. 6 could provide diverse design options to the project team. Among those, the CP combination $(15,15$, $20,20,20)$ where each number represents the number of pallets at ith zone could produce maximum number of cylinders with minimum number of pallets. In fact, this corresponds to the case with 90 pallets in Tab. 6 .

The results in Tab. 6 and Fig. 6 answer our original questions. The IZL with 90 pallets could satisfy the capacity requirement $-8.5 \mathrm{M}$, and the throughput is maximized $-10.5 \mathrm{M}$. 


\section{Further discussions}

Previous sections mentioned the analytical approach for CRP and simulation for CP. Now it would be interesting to compare two methods to evaluate the quality of the analytical solutions. Since IZL was the only feasible layout, it could work as a testbed for comparison. From Tab. 5 and Fig. 6, both CRP and CP seem to be closer. For example, the CRP at the four-lane zone is 14 while CP is 15 , and CRP at six-lane zone is 22 while CP is 20 . However, this similarity in the number of pallets may mislead readers since the CRP was computed with $8.5 \mathrm{M}$ as the target throughput while the $\mathrm{CP}$ was estimated to produce up to $10.5 \mathrm{M}$ per year. Hence, for meaningful comparison, the throughput should be synchronized. The procedure was summarized in Tab. 7.

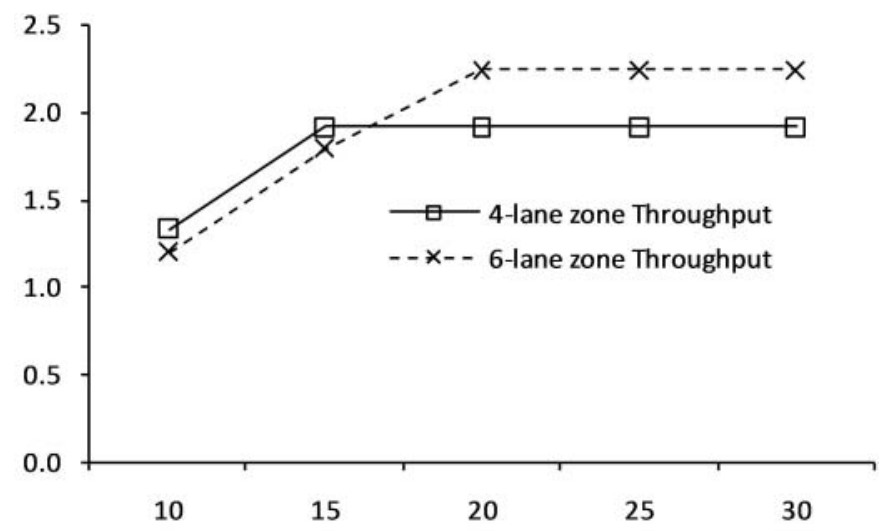

Fig. 6. Annual Throughput Per Number of Pallets

The three sub-columns under "Original CRP" represent the metrics from the current approach, rewritten from Tab. 5, where the original target delivery rate, $d_{P F O / T C O}=31$ units/min, was distributed to each zone proportionally to its number of lanes in the zone. For example, the target delivery rate at the four-lane zone, $d_{P F O / T C O}=4.73$ units $/ \mathrm{min}$, was computed by $(31$ units $/ \mathrm{min}) \times(4 / 26)$. This generated 14 pallets with $1.32 \mathrm{M}$ annual throughput (TP) that have been computed by $(4.73 \mathrm{units} / \mathrm{min}) \times(280,800 \mathrm{~min} /$ year $)$. In fact, this capacity distribution method has been consistently used in the analytical approach. However, the gap in throughput between the analytical approach under "Original CRP" and the simulation under "Simulation" tells us that this capacity distribution method was not accurate. Hence, the throughput synchronization with simulation was described in the columns under "Updated CRP". For example, in case of 4-lane zone, the annual throughput $1.9 \mathrm{M}$ corresponds to the updated target delivery rate, $d_{P F O} / T C O=6.85 \mathrm{units} / \mathrm{min}$, which generated the updated CRP, 20 pallets, with the delivery cycle time $T_{P F O / T C O}=2.89 \mathrm{~min}$. The same procedure was applied to the 6-lane zone. Compared to simulation, the analytical approach - "Updated CRP" - generated 33\% and 25\% more pallets in four-lane and six-lane zones, respectively. Note that the values for total systems - five stations - were computed in the last row.

In other words, the analytical approach may provide quick overviews for the number of pallets required without consideration of congestion caused by pallets, topology and mechanical structure of the system. However, there is no guarantee that the solution makes the system feasible. In this case study, the current analytical approach seemed to overestimate the number of pallets required.

Table 7. Comparison between CRP and CP

\begin{tabular}{c|ccc|ccc|cc|c}
\hline \multirow{2}{*}{ Type } & \multicolumn{3}{|c|}{ Original CRP } & \multicolumn{3}{c|}{ Updated CRP } & \multicolumn{2}{c}{ Simulation } & \multirow{2}{*}{ DEV\% } \\
\cline { 2 - 9 } & $d_{P F O / T C O}$ & $N_{P F O / T C O}$ & $\mathrm{TP}$ & $d_{P F O / T C O}$ & $N_{P F O / T C O}$ & $\mathrm{TP}$ & $\mathrm{CP}$ & $\mathrm{TP}$ & \\
\hline 4 lane zone & 4.73 & 14 & $1.32 \mathrm{M}$ & 6.85 & 20 & $1.9 \mathrm{M}$ & 15 & $1.9 \mathrm{M}$ & $33 \%$ \\
6 lane zone & 7.10 & 22 & $1.99 \mathrm{M}$ & 8.00 & 25 & $2.3 \mathrm{M}$ & 20 & $2.3 \mathrm{M}$ & $25 \%$ \\
Total & & 94 & $8.64 \mathrm{M}$ & & 115 & $10.5 \mathrm{M}$ & 90 & $10.5 \mathrm{M}$ & $28 \%$ \\
\hline
\end{tabular}




\section{Conclusions}

This case study handled the manufacturing process design and the capacity issue in the PFE manufacturer in North America. The vender developed an initial design of PFO and TCO stations without any quantitative modeling steps, and eventually faced the deadlock problem. Later, the project team used both an analytical approach and a simulation approach to revise and evaluate the alternatives proposed to solve this deadlock problem. The simulation approach identified that the independent zone layout could satisfy the capacity requirement by computing $\mathrm{CP}$, providing the assurance that the actual reconfiguration would generate a feasible system. However, the analytical approach to compute CRP seemed to overestimate the number of pallets without consideration of the topology and mechanical structure of the system, and it often did not guarantee the feasibility. The project team also learned that the "what-if" scenario analysis using simulation could improve the overall design process through the progressive elaboration. They also realized that an appropriate use of quantitative approaches could save the time and cost significantly particularly in the design phase of the project.

\section{References}

[1] Flexsim simulation software user guide version 3.5. 2006. Flexsim Software Products Inc.

[2] F. Abdulmalek, J. Rajgopal. Analyzing the benefits of lean manufacturing and value stream mapping via simulation: A process sector case study. International Journal of Production Economics, 2007, 107(1): 223-236.

[3] K. Duggan. Creating Mixed Model Value Stream. Productivity Press, 2002.

[4] M. Groover. Automation, Production Systems and Computer-Integrated Manufacturing. Prentice-Hall, 2001.

[5] Y. Ho, T. Liaoa. Zone design and control for vehicle collision prevention and load balancing in a zone control agv system. Computers \& Industrial Engineering, 2009, 56(1): 417-432.

[6] W. Hopp, M. Spearman. Factory Physics. McGraw-Hill Higher Education, 2008. New York.

[7] I. Lasa, C. Laburu, V. Castro. An evaluation of the value stream mapping tool. Business Process Management Journal, 2008, 14: 39-52.

[8] A. Law. Simulation Modeling and Analysis. McGraw-Hill, 2007.

[9] Y. Lian, H. Landeghem. An application of simulation and value stream mapping in lean manufacturing. Proceedings of 14th European Simulation Symposium, 2002.

[10] PMI. A guide to the project management body of knowledge. 2004. Project Management Institute Inc.

[11] K. Schwalbe. Information Technology Project Management. Course Technology, 2007. 